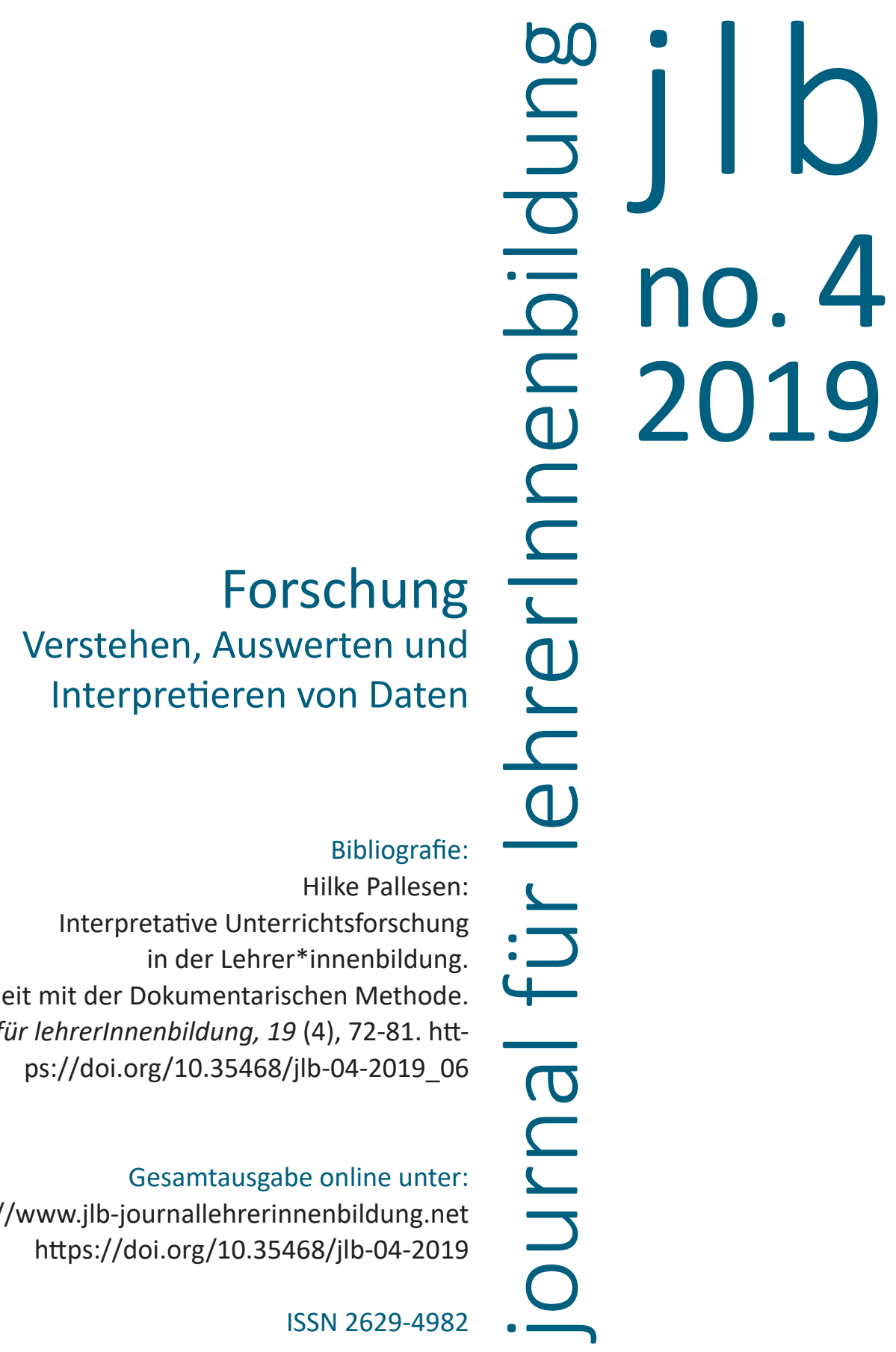


06

Hilke Pallesen

\section{Interpretative Unterrichtsforschung in der Lehrer*innenbildung. Fallarbeit mit der \\ Dokumentarischen Methode}




\section{Einleitung}

In der Lehrer*innenbildung wird der Entwicklung "fallverstehender Reflexivität" (Kolbe \& Combe, 2008, S. 877) zunehmend Bedeutsamkeit zugeschrieben. Das Lernen am Fall lässt jedoch weder standardisiertes Deuten noch routinisierte Muster der Bearbeitung zu, sondern kann nur auf der Grundlage interpretativer Verfahren vermittelt werden. Ein solches Verfahren der interpretativen Unterrichtsforschung soll im folgenden Beitrag im Zentrum stehen: die Dokumentarische Methode.

Ziel des Beitrags ist es, einen Einblick in die Seminararbeit mit der Dokumentarischen Methode in der universitären Lehrer*innenbildung zu geben und deren Potenziale und Herausforderungen zu reflektieren. Den Rahmen bildet ein kasuistisch angelegtes zweisemestriges Einführungsmodul, das alle Lehramtsstudierenden an der Universität Halle im Zuge ihres bildungswissenschaftlichen Studiums im ersten und zweiten Semester besuchen. Neben der Anbahnung einer reflexiven Haltung geht es dort v. a. darum, dass berufsrelevantes, wissenschaftliches Wissen nicht nur rezeptiv, sondern auch theoretisierend auf die Problemfälle des Berufes verwendet werden kann.

Um das im Fokus stehende kasuistisch ausgerichtete Seminarkonzept zu veranschaulichen, folgt in einem ersten Schritt der zentrale theoretische Bezug auf das sogenannte implizite Wissen, das sowohl für die Wahrnehmung unterrichtlicher Situationen als auch für dessen Gestaltung von Bedeutung ist (1), um dann in einem zweiten Schritt mit der Dokumentarischen Methode ein Verfahren vorzustellen, das eben genau diese nicht unmittelbar zugängliche Wissensebene in den Blick nimmt (2). Ein Fallbeispiel aus der Lehrer*innenbildung dient der Veranschaulichung der einzelnen Arbeitsschritte der Dokumentarischen Methode in ihrer Anwendung auf Unterrichtsprotokolle (3). Abschließend wird in einem Fazit auf Potenziale und Herausforderungen dieses Zuganges in der universitären Lehrer*innenbildung hingewiesen (4).

\section{Implizites Wissen und unterrichtliches Handeln}

Wenn es um die Beschreibung pädagogischen Handelns im Lehrer*innenberuf geht, dann wird in verschiedenen theoretischen und empirischen Ansätzen immer wieder auf die Bedeutung implizi- 
ter Wissensbestände von Lehrer*innen verwiesen (Combe \& Kolbe, 2008). Demnach beschreiben diese Vorstellungen und Ansichten, wie beispielsweise Schüler*innen wahrgenommen und anerkannt werden, wie mit Heterogenität und Leistungsbewertung, aber auch mit unterrichtlichen Krisen umgegangen wird. Bereits Lehramtsstudierende haben im Laufe ihrer Schüler*innensozialisation fest verankerte Vorstellungen darüber entwickelt, wie der Schul- und Unterrichtsbetrieb funktioniert. Diese intuitiven Ansichten und Auffassungen werden auf der Grundlage geteilter Erfahrungen erworben und lassen sich somit als habitualisiertes Wissen spezifizieren, das aber nicht unmittelbar verbalisiert werden kann (Bohnsack, 2014). Es wird in sogenannten konjunktiven Erlebnisräumen wie der Schule oder der Schulklasse angeeignet und muss aufgrund seiner hohen Beharrlichkeit, gerade in der Arbeit mit Fällen, thematisiert, irritiert und auch reflektiert werden, um so auch verschiedene Perspektiven wahr- und einnehmen zu können.

Unterricht lässt sich damit zum einen als Erfahrungsraum auffassen, der die Orientierungen der Beteiligten unterschiedlich prägen und somit auch in unterschiedlicher Weise biografisch wirksam werden kann. Zum anderen lässt sich Unterricht selbst als „Umschlagplatz und Verhandlungsort der aus den außerunterrichtlichen und außerschulischen Erfahrungsräumen mitgebrachten Aspektstrukturen" (Bonnet, 2011, S. 200) auffassen: Schüler*innen kommen mit unterschiedlichen Erfahrungshintergründen, Interessen und Vorstellungen in den Unterricht und schließen daher auch unterschiedlich an die im Unterricht präsentierten Themen und Aufgabenstellungen an. Daher lässt sich Unterricht oftmals nicht als ein gemeinsamer Erfahrungsraum rekonstruieren, denn mitunter können von der Lehrperson antizipierte Anschlüsse an den Sinnkonstruktionen der Lernenden vorbeigehen (Asbrand \& Martens, 2018).

Dieser Zusammenhang von Erfahrungshintergründen und habitualisierten Praktiken ist zentral für den Zugang der Dokumentarischen Methode. Sie fußt auf der von Mannheim entwickelten erkenntnisleitenden Unterscheidung zwischen dem explizierbaren, kommunikativen Wissen über etwas, etwa das Wissen um die verschiedenen Phasen einer Unterrichtsplanung auf der einen Seite und dem atheoretischen, konjunktiven und durch konkrete Sozialisationserfahrungen geprägten impliziten Wissen innerhalb eines Erfahrungsraumes auf der anderen Seite. Um die Unterschiede zwischen kommunikativer 
und konjunktiver Verständigung deutlich zu machen, werden zunächst zwei Analyseschritte benötigt: die formulierende und die weitaus intensivere reflektierende Interpretation. In einem letzten Schritt werden die Ergebnisse der Analyse in einer Fallbeschreibung zusammengefasst.

\section{Rekonstruktion impliziter Wissensbestände in einem Fallseminar}

Bevor im Folgenden näher auf den Einsatz der Dokumentarischen Methode in der Lehrer*innenbildung eingegangen wird, soll vorab in aller Kürze das hochschuldidaktische Verständnis des o. g. Einführungsmoduls skizziert werden.

Mit dem Ziel des Aufbaus und der Erweiterung professioneller Haltungen und theoretisierender Reflexionskompetenz, liegt der Fokus auf der Arbeit mit Fällen. Das Modul besteht aus einem Einführungsseminar, das zunächst in die Strukturmerkmale von Schule, Lehrer*innenhandeln und Unterricht einführt, einem Beobachtungspraktikum, in dem die Studierenden Protokolle zu ausgewählten Beobachtungsschwerpunkten anfertigen und einem Fallseminar, in dem diese Protokolle u. a. auch mit der Dokumentarischen Methode ausgewertet werden. Dieses Fallseminar soll nun im Folgenden für die Darstellung der Arbeit mit der Dokumentarischen Methode in der Lehrer*innenbildung als Rahmen dienen. Dabei wird zunächst davon ausgegangen, dass die Arbeit mit Fällen nur dann gelingen kann, wenn sich die Studierenden in die Anwendung dieser interpretativen Forschungsmethode umfassend einarbeiten und auch die jeweiligen sozialtheoretischen Grundlagen reflektieren können. Dazu ist es erforderlich, dass sie in den ersten Sitzungen des Fallseminars anhand von Beispielfällen in die Logik der Dokumentarischen Methode ${ }^{1}$ eingeführt werden (z. B. Schelle, Rabenstein \& Reh, 2010), um so eine differenzierte Betrachtung von unterrichtlichen Interaktionen zu ermöglichen, indem über ein mehrstufiges Verfahren der Graben zwischen kommunikativer und konjunktiver Verständigung überwunden wird.

1 Das methodische Vorgehen wurde den Ansprüchen einer forschungsbezogenen und fallorientierten Lehrer*innenbildung angepasst (insb. zum methodologischen Hintergrund Bohnsack, 2014). 
Im weiteren Verlauf des Seminars stehen dann die im Beobachtungspraktikum erstellten Protokolle im Fokus. Die Studierenden bilden nach vorab festgelegten Themenschwerpunkten (z. B. Unterrichtsanfänge, -störungen, -interaktionen) Kleingruppen und einigen sich auf ein Protokoll, das sie im weiteren Verlauf des Seminars intensiv bearbeiten. Auf diese Weise sind auch vergleichende Betrachtungen mit anderen Fällen durchaus möglich und erwünscht. Je nach Länge des Dokuments wird eine Passage ausgewählt, die für die leitende Fragestellung von Interesse ist. Als weitere Kriterien können dann beispielsweise auffällige Sprechakte oder auch metaphorische Umschreibungen herangezogen werden. Bevor die Studierenden in die Analyse einsteigen, sollen sie, um einen ersten Zugriff auf den Fall zu bekommen, dem Protokoll eine Überschrift geben, die durchaus auch aus einem Zitat bestehen kann.

Erfahrungsgemäß bereitet der Schritt der formulierenden Interpretation kaum Schwierigkeiten. Hier wird das, was in einer Unterrichtssituation wörtlich gesagt und damit thematisch wird, herausgearbeitet. Dazu teilen die Studierenden den Text in thematische Sinnabschnitte und paraphrasieren den Inhalt dieser Sinnabschnitte in zusammenfassenden Formulierungen bzw. Überschriften, um so das, was tatsächlich gesagt oder getan wurde, von dem zu unterscheiden, was möglicherweise gemeint wurde. In der fallorientierten Lehrer*innenbildung hat es sich bewährt, die Themensetzungen eher knapp zu protokollieren (siehe Fallbeispiel).

In der reflektierenden Interpretation gerät dann die Art und Weise, wie etwas gesagt wird, also wie bspw. in ein Unterrichtsthema eingeführt oder wie zwischen Lehrperson und Schüler*innen verhandelt wird, in den Fokus der Analyse. Dazu werden die zuvor herausgearbeiteten thematischen Abschnitte Schritt für Schritt durchgearbeitet. Es geht zum einen um die Art und Weise der unterrichtlichen Interaktion, und zum anderen um die Prozesshaftigkeit des unterrichtlichen Geschehens. Dabei sind zwei Prinzipien zentral: Zum einen das Prinzip einer konsequent sequenziellen Analyse, welches durch ein abschnittweises, der Vollzugs- und Diskurslogik der Unterrichtssituation folgendes Vorgehen bestimmt wird, und zum anderen das Prinzip eines komparativen Vorgehens, für das sowohl Passagen innerhalb des Protokolls als auch zwischen verschiedenen Fällen als Vergleichshorizonte hinzugezogen werden. Die Seminarleitung ist bei diesem Schritt v. a. in der Begleitung der einzelnen Kleingruppen gefordert, um darauf 
zu achten, dass voreilige Zuschreibungen und Wertungen vermieden bzw. reflektiert werden, indem ggf. auch diese Wertungen oder Alltagsvorstellungen hinterfragt werden. Um sich der reflektierenden Interpretation anzunähern, haben sich folgende Fragen an das Material als hilfreich herausgestellt, die sowohl den Inhalt als auch die Struktur der Interaktion fokussieren:

- Welche Begriffe werden benutzt/wiederholt? Welche Assoziationen lösen diese aus?

- Welchen Themen schließen sich die Beteiligten an, von welchen grenzen sie sich $a b$ ?

- Wie sprechen die beteiligten Akteur*innen miteinander? Wie stellen sie die unterrichtliche Rahmung her?

Die Ergebnisse dieser beiden Interpretationsschritte werden im Anschluss in einer Fallbeschreibung verdichtet, die die rekonstruierten Orientierungen und Sinnzuschreibungen zusammenfassend darstellen. Im Folgenden wird das Vorgehen exemplarisch anhand einer protokollierten Unterrichtsepisode zum Beobachtungsschwerpunkt Lehrer*innen-Schüler*innen-Interaktion aufgezeigt.

\section{„vielleicht ist es ja einfach unverständlich“}

\section{Protokoll}

Themen

L: Wir machen heute weiter bei den soziologischen Modellen. Was ist zu sehen? (keiner meldet sich) Ihr macht es mir heute aber auch schwer. Versucht es doch mal! Es kommt doch auf nichts an! S1, fang du bitte mal an! Du schaust so unsicher.

S1: Es ist halt so metaphorisch.

L: S2!

S2: Das ist zuviel für mich.

L: Wo ist denn jetzt das Problem? Gebt mir mal ein Feedback.

S3: Ich verstehe soziologische Modelle nicht.

S4: Naja, da ist halt ein Mann und er sitzt irgendwie in einem umgedrehten Tisch und da ist ein Wegweiser, Problemabfrage wo nichts draufsteht und er schaut so fragend irgendwie. Ich denke, er kann sich nicht entscheiden, wo er hinsoll.

S5: Aber er schwimmt auch, das sieht aus wie ein Boot und er hat ja auch ein Ruder dabei. 
L: Ihr müsst euch jetzt entscheiden. Enthaltungen gibt es nicht. Eine Deutung! Wer ist dafür? Wer dagegen? Ich nehme jeden dran. (Die Schüler*innen werden aufgefordert, für Option 1 oder 2 Handzeichen zu geben. Das Ergebnis ist unentschieden.)

S1: Können Sie nochmal die Kernaussage formulieren? (Lachen)

L: Das sollt ihr ja eigentlich machen.

S6: Ich finde es halt schwer verständlich.

L: Da würden dir hier bestimmt viele zustimmen, vielleicht ist es ja einfach unverständlich.

Im Rahmen der formulierenden Interpretation lässt sich die vorliegende Passage in vier grobe thematische Abschnitte unterteilen. Zunächst werden in dieser Unterrichtsinteraktion die Aufgabenstellung und der Umgang mit dieser thematisch. Die unterrichtlichen Sichtstrukturen folgen einem fragend-entwickelnden Modus des Unterrichtens: Die Lehrperson fragt, die Schüler*innen antworten. Die Antwort wird bewertet oder auch über das Aufrufen der nächsten Person als falsch kommentiert. Anschließend folgt im nahezu gleichen Modus eine Frage der Lehrperson nach Problemen mit der Aufgabenstellung. Auch hier reagieren die Schüler*innen auf die Anfrage, wobei die Bewertung der Antworten in der darauffolgenden Sequenz über eine Abstimmung durch die Lehrerin initiiert wird. In der vierten Passage wird schließlich eine Schülerfrage thematisch, die aber von der Lehrerin zurückgewiesen und letztendlich auch inhaltlich nicht beantwortet wird. Im Rahmen der reflektierenden Interpretation lassen sich dann über die zu bearbeitende Frage, wie etwas gesagt bzw. getan wird, v. a. Relevanzsetzungen der Lehrperson in der Interaktion rekonstruieren: Es wird schon in der ersten Passage eine Orientierung an einem Unterricht deutlich, der ,durchgezogen' wird, mitunter werden dazu auch die Ansprüche heruntergeschraubt - „es kommt ja auf nichts an." Zudem deutet sich eine Orientierung an einer persönlichen Involviertheit und damit eine Abkehr vom rollenförmigen Lehrer*innenhandeln an, wenn der Unterricht ins Stocken gerät. Fehlende Antworten werden persönlich genommen: "Ihr macht es mir aber auch schwer!“ Auch in der 2. Passage werden die Schüler*innen über die Problemabfrage durch die Lehrerin erneut in die Verantwortung für einen rei- 
bungslosen Unterricht gezogen. Diese nehmen die zugewiesene Rolle als Rettungsanker und Unterstützende der Lehrperson auch an, zeigen damit eine hohe Kooperationsbereitschaft und halten die unterrichtliche Ordnung aufrecht, indem sie sich nicht etwa der Antwort verweigern, sondern versuchen, zu einer Lösung zu gelangen.

Letztendlich wird in der dritten Passage von der Lehrkraft die Option einer Abstimmung gewählt, die fernab von der Sache zwar den Unterricht aufrechterhält, denn auch hier nehmen die Schüler*innen bereitwillig daran teil, letztendlich aber nicht zu einem Ergebnis führt. In der vierten Passage lässt sich wiederum eine hohe Bereitschaft der Lernenden aufzeigen, mit der Aufgabe zu beginnen. Hier wird die Fragerichtung gewissermaßen über die Schüler*innenfrage ,umgedreht'. Darüber wird deutlich, dass sie dem Gegenstand und dem Wissenserwerb eine hohe Relevanz zuschreiben, was sich letztendlich auch darüber ausdrückt, dass sie auch Hilfegesuche formulieren: „Das ist zu viel für mich".

Das Ziel der reflektierenden Interpretation besteht darin, die vorliegende Situation zu verstehen, die Beurteilung der Unterrichtsqualität steht nicht im Fokus einer rekonstruktiven Annäherung an das Material. Dennoch deuten die Inkonsistenzen in der Interaktion, die fehlenden Bezüge der Lehrperson auf die Antworten und Hilfegesuche der Schüler*innen sowie die Abgabe der formalen Verantwortung für den Unterricht und schließlich die offensichtlich nicht zu lösende Aufgabe („vielleicht ist es ja auch nicht verständlich“) darauf hin, dass hier die Voraussetzungen der Lernenden nicht mit dem geplanten Unterrichtsgegenstand in ein produktives Verhältnis gesetzt werden können (Helsper, 2016, S. 119ff). Mit der Anbindung des Falles an einen theoretischen Diskurs, wie z. B. an den des strukturtheoretischen Professionalisierungsansatzes nach Oevermann (z. B. 1996, 2002, 2008) wird die Arbeit am Fall abgeschlossen.

\section{Fazit}

Die Dokumentarische Methode eignet sich zur Auswertung einer Vielzahl unterschiedlicher qualitativer Daten. Ursprünglich wurde sie zur Rekonstruktion von Gruppendiskussionen mit Jugendlichen entwickelt, um darüber kollektiv geteilte Orientierungen zu dokumentieren, inzwischen wird sie auch für Interviews, Bilder und Videos, aber auch für 
Dokumente und Beobachtungsprotokolle eingesetzt. Der verlangsamte Blick auf das unterrichtliche Geschehen dient der Reflexionssteigerung. Die Arbeit mit der Dokumentarischen Methode ist aber auch mit Herausforderungen verbunden: Die kleinschrittige Interpretation von Texten erfordert eine Rezeptionshaltung, die der gewohnten Leserichtung Studierender oftmals zuwiderläuft. Die intensive Textarbeit und auch das Gebot, voreilige Schlüsse zurückzustellen, kann zunächst Irritationen auslösen. Diese lassen sich in der Seminararbeit auch kaum vermeiden, aber dennoch produktiv bearbeiten, indem anhand des Fallmaterials deutlich gemacht wird, dass Unterricht unter dieser sozialwissenschaftlichen Perspektive nicht von didaktischen Vorgaben oder von den guten Absichten der Lehrperson heraus gedacht wird, der beispielsweise ein abwechslungsreiches Unterrichten unterstellt wird, sondern dass sich Unterricht aus einer sinnkonstruierenden Forschungsperspektive vielmehr als ein soziales Geschehen zeigt, das auf empirischen Daten beruhend Zusammenhänge aufdeckt, die mitunter auch von den Intentionen der Beteiligten abweichen.

Mit der bereits eingangs aufgeworfenen zentralen Annahme, dass gerade Reflexivität als unstrittiges Element pädagogischer Professionalität gilt, wird darüber auch der Anspruch der Lehrer*innenbildung deutlich, reflexive und hermeneutische Kompetenzen anzubahnen, die einerseits mit dem Erwerb und der Anwendung expliziten Wissens verbunden sind, andererseits aber auch das implizite Wissen zu reflektieren vermögen, um zu vermeiden, dass das Erfahrungswissen „nicht nur Einübung in zukünftiges Wissen ist" (Combe \& Kolbe, 2008, S. 870). Denn "nur ein reflexiver Umgang mit den eigenen, lebensgeschichtlich vorgängig erworbenen Dispositionen sichert in der Lehrer*innenbildung, dass diese nicht undurchschaut in die Entwicklung eigener Muster und Routinen Eingang finden“" (ebd., S. 892). Gerade hier bietet ein sinnverstehender, rekonstruktiver und fallbezogener Zugang die Grundlage für eine reflexive Auseinandersetzung mit Dokumenten und Ausdrucksformen aus der Schul- und Unterrichtspraxis, die auf diese Weise handlungsdruckentlastet betrachtet werden können. So lässt sich gerade für die o. g. unterrichtliche Interaktion über die unmittelbare Sichtstruktur hinaus, über die zwar eine unterrichtliche Ordnung hergestellt wird, rekonstruieren, dass an den Voraussetzungen der Schüler*innen vorbei agiert wird. Ziel ist es, darüber berufsrelevantes wissenschaftliches Wissen nicht nur rezeptiv anzueignen, sondern es mit Blick auf Praxis nachzuerzeugen und 
theoretisierend zu verwenden. Gerade der Umgang mit einzelnen, konkreten pädagogischen Situationen sensibilisiert für die Komplexität von Unterricht, was letztendlich auch dazu beitragen kann, in realen Situationen handlungsfähig zu bleiben.

\section{Literatur}

Asbrand, B. \& Martens, M. (Hrsg.). (2018). Dokumentarische Unterrichtsforschung. Wiesbaden: VS.

Bohnsack, R. (2014). Rekonstruktive Sozialforschung. Einführung in qualitative Methoden. Opladen \& Toronto: Barbara Budrich.

Bonnet, A. (2011). Erfahrung, Interaktion, Bildung. In W. Meseth, M. Proske \& F.-O. Radtke (Hrsg.), Unterrichtstheorien in Forschung und Lehre (S. 189-208). Bad Heilbrunn: Julius Klinkhardt.

Combe, A. \& Kolbe, F.-U. (2008). Lehrerprofessionalität: Wissen, Können, Handeln. In W. Helsper \& J. Böhme (Hrsg.), Handbuch Schulforschung (S. 857-875). Wiesbaden: VS.

Helsper, W. (2016). Lehrerprofessionalität - der strukturtheoretische Ansatz. In M. Rothland (Hrsg.), Beruf Lehrer/Lehrerin (S. 103-125). Waxmann: Münster.

Kolbe, F.-U. \& Combe, A. (2008). Lehrerbildung. In W. Helsper \& J. Böhme (Hrsg.), Handbuch Schulforschung (S. 877-901). Wiesbaden: VS.

Oevermann, U. (1996). Theoretische Skizze einer revidierten Theorie professionalisierten Handelns. In A. Combe, W. Helsper (Hrsg.), Pädagogische Professionalität. Eine Untersuchung zum Typus pädagogischen Handelns (S. 70-182). Frankfurt a. M.: Suhrkamp.

Oevermann, U. (2002). Professionalisierungsbedürftigkeit und Professionalisiertheit pädagogischen Handelns. In M. Kraul, W. Marotzki \& C. Schweppe (Hrsg.), Biographie und Profession (S. 12-63). Bad Heilbrunn: Klinkhardt.

Oevermann, U. (2008) Profession contra Organisation? Strukturtheoretische Perspektiven zum Verhältnis von Organisation und Profession in der Schule. In W. Helsper, S. Busse, M. Hummrich \& R.-T. Kramer (Hrsg.), Pädagogische Professionalität in Organisationen. Neue Verhältnisbestimmungen am Beispiel der Schule (S. 56-77). Springer: Wiesbaden.

Schelle, C., Rabenstein, K. \& Reh, S. (2010). Unterricht als Interaktion. Ein Fallbuch für die Lehrerbildung. Bad Heilbrunn: Julius Klinkhardt.

Hilke Pallesen, Dr., wiss. Mitarbeiterin im Arbeitsbereich Schulpädagogik/Schulforschung an der MLU Halle-Wittenberg. Arbeitsschwerpunkte: Schul- und Fachkulturforschung, Professions- und Lehrer*innenbildungsforschung

hilke.pallesen@paedagogik.uni-halle.de

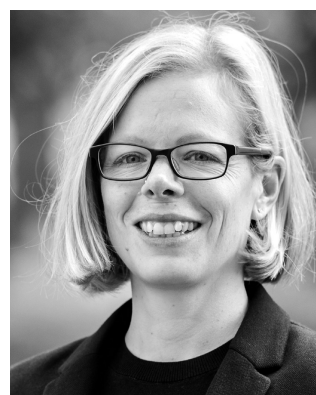

